Fachdental Awards 2015

\title{
Hightech für ein gesundes Lächeln
}

Die Aussteller der Fachdental Leipzig und der Fachdental Südwest schicken in diesem Jahr erstmals ihre innovativsten Ideen, aktuellen Prototypen und Produkteinführungen gegeneinander ins Rennen. Die Besucher entscheiden, welche Teilnehmer hinsichtlich Innovationsgrad, Nutzen und Design am meisten überzeugen und prämiert werden sollen. Mit der Auszeichnung möchte die Messe Stuttgart die hohe Innovationskraft der Branche darstellen. Das Teilnehmerfeld repräsentiert mit Medizintechnik-, Material,- Laborausstattungs- und Softwareanbietern die gesamte Branche.

Während beider Messen haben Besucher die Möglichkeit, eingereichte Produkte auf dem „Marktplatz der Ideen“ kritisch zu prüfen und dann an Terminals abstimmen. Bereits vorab präsentieren sich die Kandidaten online auf einer Voting-Seite, auf der ebenfalls abgestimmt werden kann. Die bisherigen Kandidaten für die 1. Fachdental Awards sind Dürr Dental AG, ic med $\mathrm{GmbH}$, Ivoclar Vivadent $\mathrm{GmbH}$, KaVo Dental GmbH, Kuraray Europe $\mathrm{GmbH}$, Loser \& Co $\mathrm{GmbH}$, orangedental $\mathrm{GmbH} \&$ Co. KG, Schick GmbH. Unter allen abstimmenden Besuchern wird in Leipzig und in Stuttgart eine Applewatch Sport verlost.

Nach einer Pressemitteilung der

Landesmesse Stuttgart GmbH, Stuttgart 